\title{
Independent Modulation of the Phase and Amplitude of Electromagnetic Waves Based on the Phase Change of $\mathrm{VO}_{2}$ and Mode Coupling
}

\author{
Tianrui Pan, Yuan Pei, Maosheng Wang, Xiaojuan Xie, Wanxia Huang*
}

School of Physics and Electronic Information \& Anhui Province Key Laboratory of Optoelectronic Materials Science and Technology, Anhui Normal University, Wuhu, China

Email address:

kate@mail.ahnu.edu.cn (Wanxia Huang)

${ }^{*}$ Corresponding author

\section{To cite this article:}

Tianrui Pan, Yuan Pei, Maosheng Wang, Xiaojuan Xie, Wanxia Huang. Independent Modulation of the Phase and Amplitude of Electromagnetic Waves Based on the Phase Change of $\mathrm{VO}_{2}$ and Mode Coupling. American Journal of Physics and Applications. Vol. 9, No. 4, 2021, pp. 88-93. doi: 10.11648/j.ajpa.20210904.13

Received: July 11, 2021; Accepted: August 2, 2021; Published: August 12, 2021

\begin{abstract}
Metasurfaces in a metal-semiconductor-metal configuration have been studied in multiple contexts, including perfect absorption and phase modulation. Nevertheless, limit progress has been achieved in independent phase modulation. To study further in this work, a metasurface composed of an $\mathrm{Au}$ film/ $\mathrm{VO}_{2}$ film/ $\mathrm{Au}$ patch array with square holes was proposed in this paper. Through simulating and analysing the optical properties, simulated results indicated that an extremely switchable function can be realized by modulating the phase transition of $\mathrm{VO}_{2}$, when $\mathrm{VO}_{2}$ was in the metal (semiconductor) phase, the whole structure represented as ON (OFF) state. Additionally, the efficient modulation depth is approximately $99.6 \%$ for the y-polarization at a wavelength of $2.019 \mu \mathrm{m}$. What's more, by modulating symmetry-breaking of the structure or polarization, an extreme reflection phase change can been applied. As for the former, by adjusting the asymmetry degreed, the reflection phase can change from less than $180^{\circ}$ to nearly $360^{\circ}$, and for the latter, the adjustment in polarization resulted in a phase change of nearly $180^{\circ}$ for the x-polarization and nearly $360^{\circ}$ for the y-polarization. While the amplitude remained almost constant at the corresponding wavelength. That is to say, an independent regulation of amplitude and phase was accomplished. And a two-mode one-port temporal coupled mode theory supported by full-wave simulations can explain the underlying physics of the designed independent phase modulation. The research findings mentioned above established the possibility for plasmonic integration as well as the design of multi-functional devices such as gradient metasurfaces and temperature-controlled switches.
\end{abstract}

Keywords: Metasurfaces, Temporal Coupled Mode Theory, Vanadium Dioxide, Optical Switching

\section{Introduction}

The semiconductor-metal transition (SMT) of vanadium dioxide $\left(\mathrm{VO}_{2}\right)$ occurs when external parameters, including electric field, magnetic field, light, temperature and stress, reach critical values. In addition to being applied in other approaches for achieving the phase transition of $\mathrm{VO}_{2}[1-4], \mathrm{VO}_{2}$ has been combined with surface plasmons to achieve numerous dynamically tunable functions. For example, Xu et al. fabricated silver nanoparticles on a $\mathrm{VO}_{2}$ film and observed that the localized surface plasmon resonant band exhibited a temperature dependence ranging from $30^{\circ} \mathrm{C}$ to $80^{\circ} \mathrm{C}$ [5]. Wei and colleagues investigated new aspects of SMT in single-domain $\mathrm{VO}_{2}$ nanobeams [6]. Yan et al. designed $\mathrm{VO}_{2}$ materials for broadband absorption of nonlinear circularly polarized transformed metasurfaces in the terahertz band [7]. Furthermore, various applications for tunable devices have also emerged. Metamaterial devices with far-infrared resonance frequencies were dynamically tuned [8]. Huang et al. also implemented a temperature-controlled switch in the near-infrared band using $\mathrm{VO}_{2}$ [9]. Ooi and colleagues designed an ultra-compact $\mathrm{VO}_{2}$ two-mode plasmon-waveguide electroabsorption regulator with a switching regulation depth of $10 \mathrm{~dB}$, and this high-performance plasmon regulator may be critical to integrating optoelectronic circuits in next-generation chip technology [10]. In 2018, a frequency-tunable metasurface filter 
for the control of $\mathrm{VO}_{2}$-reconfigured optical-phase terahertz polarization was implemented [11]. Wang et al. designed a thermally switchable terahertz wavefront metasurface modulator based on $\mathrm{VO}_{2}$ for the SMT [12].

Researchers have proposed combining phase transition materials with surface plasmons. For example, Zhou and colleagues developed the phase and amplitude spectra of a sandwich-reflection system of metal film/ dielectric (graphene)/ metal nanopatterns using one-mode and one-port temporal coupled-mode theory (tCMT) [13, 14]. They reported that the dependent modulation of amplitude and phase could be achieved through the modulation of the absorption loss of graphene. However, the dependent modulation of phase and amplitude is unable to independently modulate electromagnetic waves [15].

In this paper, the metasurfaces of a metal film, a $\mathrm{VO}_{2}$ film, and a rectangular metal nanoparticle with a square hole were designed, and their optical responses were evaluated using numerical simulations. The designed metasurfaces improved previously reported temperature-controlled switching function. After the adjustment of the relative position of the square hole relative to the rectangular particle, the reflection phase for the $x$-polarization changed from nearly $360^{\circ}$ to $<180^{\circ}$, and the reflection amplitude remained constant. As a result, an independent regulation of phase was achieved. For the $y$-polarization, a change from $<180^{\circ}$ to nearly $360^{\circ}$ also occurred. After the semiconductor percentage was adjusted, reflection intensity changed substantially and phase remained constant. Consequently, the independent modulation of phase and amplitude was achieved. And the simulation results of two-mode and one-port tCMT was also reported. These results may aid in the design of multifunctional devices, phase gradient metasurfaces, and temperature-controlled switches.

\section{Material and Methods}

\subsection{Structural Design and Numerical Simulation}

(a)

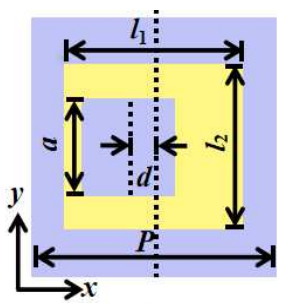

(b)

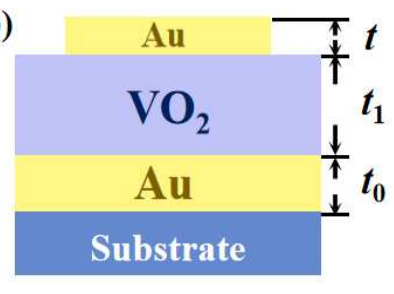

(c)

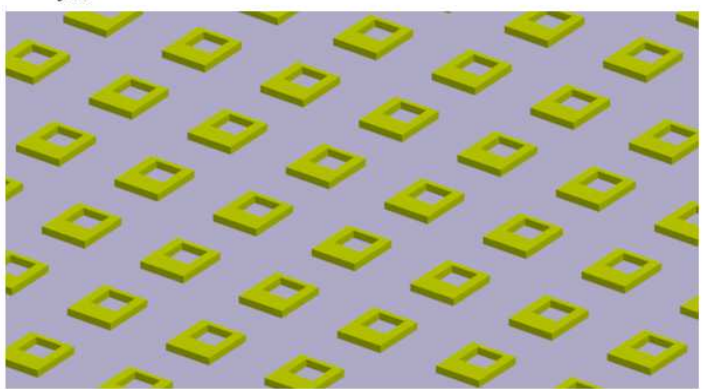

Figure 1. (Color online) (a-b) Schematic of the structural parameters of the unit cell. (c) Schematic of the metasurface.
A schematic of the metasurfaces is illustrated in Figure 1. In Figure 1(a,b), respectively, the top and side views of the unit cell are reported. A symmetrically broken nanostructure of $\mathrm{Au}$ thin film $\left(t_{0}=50 \mathrm{~nm}\right) / \mathrm{VO}_{2}$ film $\left(t_{1}=150 \mathrm{~nm}\right) /$ rectangular $\mathrm{Au}$ patches $(t=30 \mathrm{~nm})$ of size $540 \mathrm{~nm} \times 470 \mathrm{~nm}$ arranged in a square lattice with a periodicity of $750 \mathrm{~nm}$ was designed. Each Au patch was drilled with a $280 \mathrm{~nm} \times 280 \mathrm{~nm}$ air hole with the center displaced from the patch center by symmetry breaking in the metasurface. The asymmetry degree was defined as $d$. The Au thin film with a thickness $\left(t_{0}\right)$ of $50 \mathrm{~nm}$ was grown on a quartz substrate, and the $\mathrm{VO}_{2}$ film with a thickness $\left(t_{1}\right)$ of $150 \mathrm{~nm}$ was grown on an $\mathrm{Au}$ film. The thickness of the top Au patch array was $t=30 \mathrm{~nm}$. To investigate the optical properties of the metasurface, numerical simulations were performed using the finite-difference time-domain method. In the numerical simulation, the refractive indices of the substrate and air were set to 1.5 and 1, respectively, and the $\mathrm{Au}$ film was modeled using the Drude model as follows: $\varepsilon_{A u}=1-\omega_{p}^{2} / \omega(\omega+i \gamma)$, where $\omega_{p}=1.374 \times 1016 \mathrm{rad} / \mathrm{s}$ was the plasmon frequency and $\gamma=1.224 \times 1014 \mathrm{rad} / \mathrm{s}$ was the collision frequency [16]. The dielectric constant of the metal phase ( $\mathrm{M}$ phase) and semiconductor phase ( $\mathrm{S}$ phase) of $\mathrm{VO}_{2}$ were obtained according to Smith's research [17].

\subsection{Optical Properties}

Figure 2 reports the simulated reflection intensity spectra and phase spectra for the structural parameter $d=80 \mathrm{~nm}$, where Figure 2(a,b) [or (c, d)] correspond to the reflection intensity spectra and phase spectra for $x$ - (or $y$-) polarization, respectively. Irrespective of $x$ - or $y$ - polarization, the structure remained in a metal/semiconductor/metal patch state when $\mathrm{VO}_{2}$ was in the $\mathrm{S}$ phase. A dip in the reflection spectra appeared at approximately 2 $\mu \mathrm{m}$ because of magnetic resonance behavior at that wavelength. This represented the OFF state. When $\mathrm{VO}_{2}$ was in the $\mathrm{M}$ phase, the entire structure comprised as a metal film with a strong reflection and simulated reflection intensity of approximately 0.78 , which represented the ON state. To quantify the switching performance of our metasurface, the modulation depth (MOD) of switching was reported [18]:

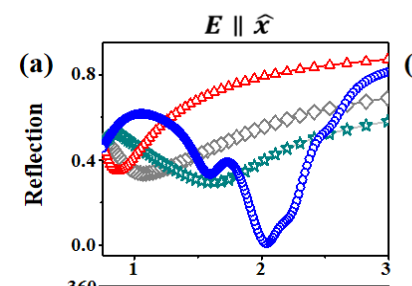

\section{(b)}
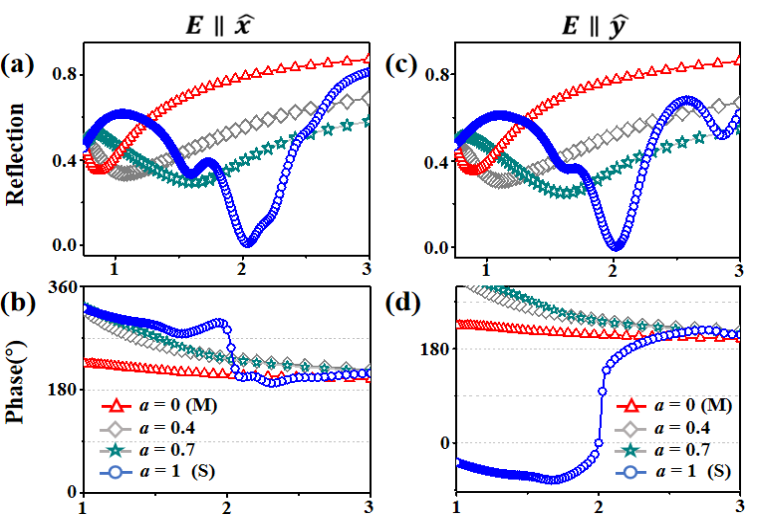

(d)

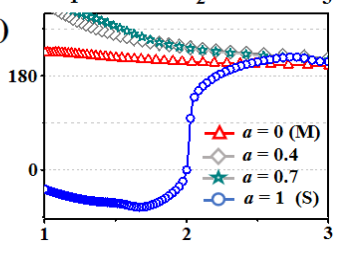

Wavelength $(\mu \mathrm{m})$

Figure 2. (Color online) Intensity $(a, c)$ and phase $(b, d)$ spectra of the metasurfaces $(d=80 \mathrm{~nm})$ with different values of a (the percentage of the $S$ phase in $\mathrm{VO}_{2}$ film) illuminated by normally incident lights with $x$-polarization $(a, b)$ and $y$-polarization $(c, d)$. The red open triangles, gray open rhombuses, glaucous open stars, and blue open circles correspond to $a=0$ (M phase), 0.4, 0.7 , and 1 (S phase), respectively. 


$$
\mathrm{MOD}=\frac{R_{\max }-R_{\min }}{R_{\max }+R_{\min }}
$$

Where $R_{\max }$ and $R_{\min }$ represent the reflection intensity of the $\mathrm{M}$ and the $\mathrm{S}$ phase at a fixed wavelength, respectively. For the $x$-polarization, $R_{\max }$ and $R_{\min }$ were taken as 0.7944 and 0.0083 at a wavelength of $2.031 \mu \mathrm{m}$, which corresponded to a MOD of approximately $97.93 \%$. For the $y$-polarization, $R_{\max }$ and $R_{\min }$ were taken as 0.777 and 0.0015 at a wavelength of $2.019 \mu \mathrm{m}$, which corresponded to a MOD of about $99.61 \%$. Consequently, the metasurface exhibited favorable switching performance, especially for the $y$-polarized light, which achieved a nearly ideal state based on the expression for the absorption factor $A$ :

$$
A=1-R-T
$$

where $T$ denotes transmission intensity. Because the thickness of the lowest metal floor was $50 \mathrm{~nm}$, the transmission in the near-infrared band was nearly zero. Therefore, in the S phase, $A_{x}=0.992$ was at a wavelength of $2.031 \mu \mathrm{m}$ for the $x$-polarization, and $A_{y}=0.999$ was at a wavelength of $2.019 \mu \mathrm{m}$ for the $y$-polarization. When $\mathrm{VO}_{2}$ was in the $\mathrm{S}$ phase, the metasurface exhibited perfect absorption. As depicted in Figures. 2(b, d), when $\mathrm{VO}_{2}$ was in the $\mathrm{M}$ phase, the phases of the two polarizations were nearly identical because the top and bottom metal layers were short-circuited. When $\mathrm{VO}_{2}$ was in the $\mathrm{S}$ phase, the phase change was less than $180^{\circ}$ at approximately $2 \mu \mathrm{m}$ for the $x$-polarization; however, for the $y$-polarization, the phase change was approximately $360^{\circ}$. Phase change was achieved by altering the polarization.

According to the classical effective medium theory [19], the shift in $\mathrm{VO}_{2}$ from the $\mathrm{S}$ phase to the $\mathrm{M}$ phase is a gradual process that occurs as temperature rises. Therefore, we also simulated the dielectric constant exhibited by the structure for varying proportions of the $\mathrm{S}$ phase in the structure. The percentage of the $\mathrm{S}$ phase in the $\mathrm{VO}_{2}$ film was set as $a$, and the dielectric constant of the $\mathrm{VO}_{2}$ film was set as $\varepsilon$ :

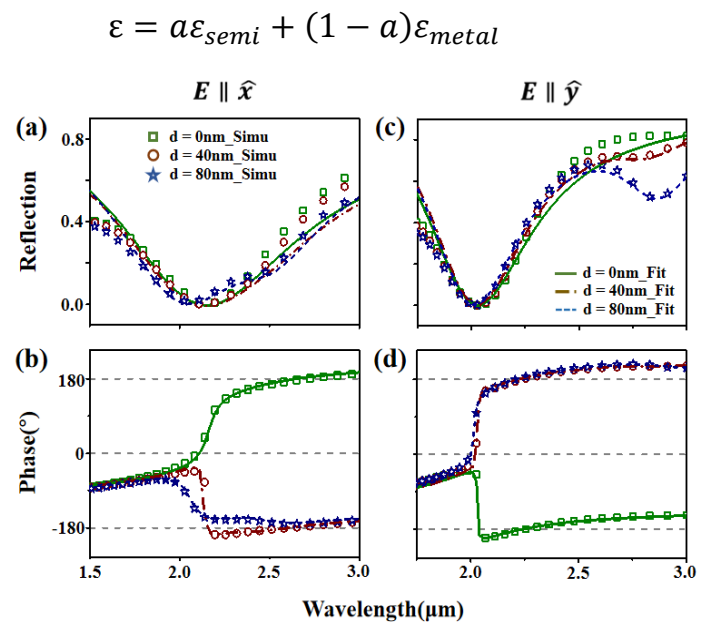

Figure 3. (Color online) Intensity $(a, c)$ and phase $(b, d)$ spectra of metasurfaces with different values of dilluminated by normally incident lights under $x$ - and y-polarization ( $a, b$ and $c, d$, respectively). The simulation results were $d=0 \mathrm{~nm}, 40 \mathrm{~nm}$, and $80 \mathrm{~nm}$ for the green olive-colored open squares, red wine-colored open circles, and navy open stars, respectively. The theoretical analysis results correspond to the olive solid curve, wine dash-dot curve, and navy short-dash curve, respectively. where $\varepsilon_{\text {semi }}$ is the dielectric constant of the $\mathrm{S}$ phase and $\varepsilon_{\text {metal }}$ is that of the $\mathrm{M}$ phase. The simulation results are reported in Figure 2 for $a$ values of 0.4 and 0.7. As indicated in Figures. 2(a, c), as $a$ increases, the reflection dip indicates a clear red shift. These results suggest a potential application of designed metasurface for filters. Additionally, through the adjustment of the a value, a substantial change in reflection intensity occurred at wavelengths of $2.075 \mu \mathrm{m}$ and $2.109 \mu \mathrm{m}$ for $\mathrm{x}$ - and $\mathrm{y}$-polarization, respectively, and the phase change remained nearly constant. At a wavelength of approximately 2 $\mu \mathrm{m}$, the adjustment in polarization resulted in a phase change of nearly $180^{\circ}$ for the x-polarization and nearly $360^{\circ}$ for the $y$-polarization, but reflection intensity remained constant. In sum, the designed structure achieved the independent regulation of amplitude and phase.

To investigate additional properties of the metasurface, research also focused on simulating the reflection intensity spectra and phase spectra in steps of $10 \mathrm{~nm}$ from $d=0 \mathrm{~nm}$ to 80 $\mathrm{nm}$ for two polarizations with $\mathrm{VO}_{2}$ in the $\mathrm{S}$ phase and the $\mathrm{M}$ phase, respectively. The simulation results indicated that the reflection intensity and phase remained constant in both polarization directions when $\mathrm{VO}_{2}$ was in the $\mathrm{M}$ phase and the asymmetry $d$ varied (Figure 2). When $\mathrm{VO}_{2}$ was in the $\mathrm{S}$ phase, selecting $d$ values of 0,40 , and $80 \mathrm{~nm}$. The normal incident plane wave, the reflection intensity, and phase spectra are depicted in Figure 3. At a wavelength of $2.031 \mu \mathrm{m}$, the phase changes of the metasurface at $d=40 \mathrm{~nm}$ and $80 \mathrm{~nm}$ were $<180^{\circ}$ for the x-polarization; however, the phase change of the metasurface at $d=0 \mathrm{~nm}$ was approximately $360^{\circ}$. For the $\mathrm{y}$-polarization, at a wavelength of $2.019 \mu \mathrm{m}$, the phase change of the metasurface at $d=0 \mathrm{~nm}$ was $<180^{\circ}$; however, the phase changes of that at $d=40 \mathrm{~nm}$ and $80 \mathrm{~nm}$ were approximately $360^{\circ}$. The reflection intensity spectra remained nearly constant at the corresponding wavelength for the two polarizations. The results indicated that symmetry-breaking of the structure significantly altered the reflection phase, but the amplitude at the corresponding wavelength remained nearly unaffected. Through the adjustment of the asymmetry degree $d$, the reflection phase increased from less than $180^{\circ}$ to nearly $360^{\circ}$ in the $1.5-2.5 \mu \mathrm{m}$ range, and the amplitude remained almost constant. Consequently, the independent modulation of phase and amplitude were achieved.

\section{Results and Discussion}

To examine the underlying physical mechanisms described by the findings, the field intensity of the symmetric $(d=0 \mathrm{~nm})$ structure under higher-order waveguide modes 1 and 2 was simulated, as well. As depicted in Figure 4(a,b), for the $x$-polarization, dip A occurred at $2.08 \mu \mathrm{m}$ and peak B occurred at $2.22 \mu \mathrm{m}$; however, for the $y$-polarization, dip C occurred at $2.03 \mu \mathrm{m}$ and peak D occurred at $2.74 \mu \mathrm{m}$. To assess the physical mechanisms of these peaks and dips, vector distribution patterns of current density were simulated and depicted in Figure 4(c-f). Figure 4(c) reports the current density distribution of dip A: the current was primarily concentrated at the Au patch's up and down arms and behaved as an electric dipole; it can be regarded as being in a bright 
mode (broad resonance). Figure 4(d) reports the current density distribution of peak B: the current was primarily concentrated at the Au patch's four inner corners, and the current direction at opposite sides were parallel. However, the current direction at diagonal sides were antiparallel and behaved as an electric quadrupole; the current represented a dark mode (narrow resonance). The asymmetric line of the reflection spectra was defined as the interference between a spectrally overlapping broad resonance and a narrow discrete resonance. As reported in Figure 4(e), for the y-polarization, the current in dip $\mathrm{C}$ was primarily concentrated at the $\mathrm{Au}$ patch's left and right arms and behaved as an electric dipole, which represented a bright mode (broad resonance). The current in peak $\mathrm{D}$ was primarily concentrated on the $\mathrm{Au}$ patch's four arms, and the current direction at opposite arms were antiparallel. This current behaved as an electric quadrupole, representing a dark mode (narrow resonance). The coherent superposition of the two modes resulted in an asymmetric linear dip at approximately $2.86 \mu \mathrm{m}$ in the asymmetric structure. This superposition of the two nodes is commonly cited as leading to Fano resonance [20, 21]. When the center of the square hole and the center of the rectangular particle do not coincide $(d \neq 0 \mathrm{~nm})$, the structural symmetry dissolves and coupling occurs between the dipole and quadrupole of the rectangular particle. This quadrupole-to-dipole action has a substantial modulating effect on the reflection phase.

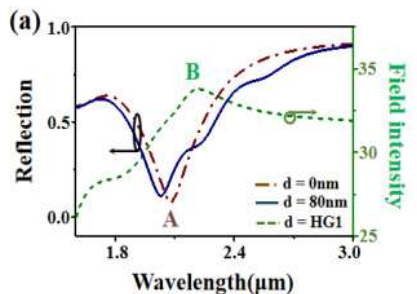

(c)

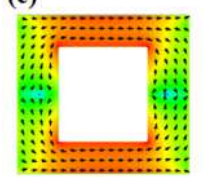

A

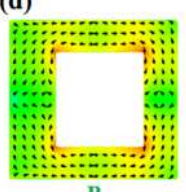

B

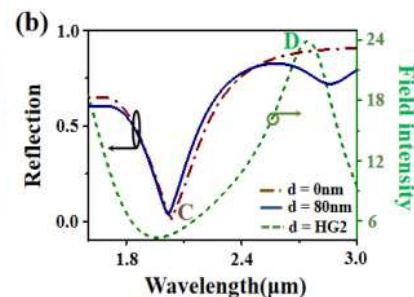

(f)

\begin{abstract}
(e)
\end{abstract}

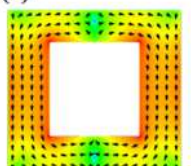

C

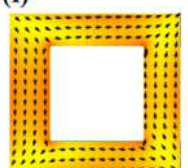

D
Figure 4. (Color online) Simulated reflectance spectra of the metasurface of the $d=0 \mathrm{~nm}$ (wine dash-dot curve) and $d=80 \mathrm{~nm}$ (navy solid curve) structures for the x-polarized (a) and y-polarized (b) normal light incidence and field intensity of the $d=0 \mathrm{~nm}$ (green olive-colored short-dash curve) structures under higher-order waveguide modes 1 (a) and 2 (b). (c-f) Simulated current density vector distribution corresponding to dip $A$, peak $B$, dip $C$, and peak $D$, respectively.

The two-mode and two-port tCMT explained many elements of the transmissive system [20, 22, 23], whereas the two-mode and one-port tCMT was less instructive [24]. We introduce the following two-mode and one-port tCMT under coupled basis vectors to explain the results of our simulation by employing the kinetic equation:

$$
\frac{d}{d t}\left(\begin{array}{l}
a_{p} \\
a_{q}
\end{array}\right)=-i\left(\begin{array}{cc}
\omega_{p} & 0 \\
0 & \omega_{q}
\end{array}\right)\left(\begin{array}{l}
a_{p} \\
a_{q}
\end{array}\right)-\left(\begin{array}{cc}
\gamma_{p}+\gamma_{p}^{\prime} & \gamma_{p q} \\
\gamma_{p q} & \gamma_{q}+\gamma_{q}^{\prime}
\end{array}\right)\left(\begin{array}{l}
a_{p} \\
a_{q}
\end{array}\right)+
$$

$$
\left(\begin{array}{l}
d_{p} \\
d_{q}
\end{array}\right) S^{+}
$$

where the dressed bright mode and dressed dark mode after near-field coupling corresponded to modes $p$ and $q$, respectively. The values $a_{\alpha}, \omega_{\alpha}, \gamma_{\alpha}, \gamma_{\alpha}^{\prime}(\alpha=p, q)$ were the complex amplitude, circular frequency, radiation, and absorption losses for the $\alpha$-th dressed modes, respectively. The value $\gamma_{p q}$ was the far-field coupling between two dressed modes. The value $d_{\alpha}$ denotes the coupling coefficient between the port and the $\alpha$-th mode. These parameters were associated with those of uncoupled basis vectors (pure dipole and pure quadrupole) through a representation transformation [20]. $S^{+}$and $S^{-}$corresponded to the incoming and outgoing ports, respectively. The expression of the outgoing wave is given as

$$
S^{-}=C S^{+}+\left(\begin{array}{ll}
d_{p} & d_{q}
\end{array}\right)\left(\begin{array}{l}
a_{p} \\
a_{q}
\end{array}\right)
$$

where $\mathrm{C}$ represented the background consisting of the metal and $\mathrm{VO}_{2}$ films. According to the characteristics of our structure, $\mathrm{C}$ was thus taken as -1 . After accounting for energy conservation, then obtain

$$
\left(\begin{array}{ll}
d_{p} & d_{q}
\end{array}\right)^{\dagger}\left(\begin{array}{ll}
d_{p} & d_{q}
\end{array}\right)=2\left(\begin{array}{cc}
\gamma_{p} & \gamma_{p q} \\
\gamma_{p q} & \gamma_{q}
\end{array}\right)
$$

Where " $\uparrow "$ denotes the complex conjugate transpose of a matrix. According to the time-reversal symmetry, then obtain

$$
\begin{aligned}
C\left(d_{p} \quad d_{q}\right)^{*} & =-\left(\begin{array}{ll}
d_{p} & d_{q}
\end{array}\right) \\
\mathrm{CC}^{*} & =1
\end{aligned}
$$

By calculating Eqs. (6) and (7), then obtain

$$
\begin{gathered}
d_{\alpha}=d_{\alpha}{ }^{*},\left|d_{\alpha}\right|=\sqrt{2 \gamma_{\alpha}}(\alpha=p, q) \\
\gamma_{p q}=\frac{1}{2} d_{p}{ }^{*} d_{q}=\eta \sqrt{\gamma_{p} \gamma_{q}}
\end{gathered}
$$

which $\eta$ is related to the phase of the coupling coefficient of the two modes to the port. Because both modes radiated outwards on the same plane, so they can be assumed that were on the same phase; and $\eta$ equaled 1.

After Eqs. (4) and (5) were solved and combined with Eqs. (9) and (10), the reflection coefficient $r$ under the coupled basis vector was as follows:

$$
r \approx-1+2\left(\frac{\gamma_{p}}{\mathrm{w}_{p}}+\frac{\gamma_{q}}{\mathrm{w}_{q}}-2 \frac{\gamma_{q} \gamma_{p}}{\mathrm{w}_{q} \mathrm{w}_{p}}\right) /\left(1-\frac{\gamma_{q} \gamma_{p}}{\mathrm{w}_{q} \mathrm{w}_{p}}\right)
$$

where $\mathrm{W}_{q}=-i\left(\omega-\omega_{\alpha}\right)+\gamma_{\alpha}+\gamma_{\alpha}^{\prime}(\alpha=p, q)$. The first two terms of the numerator describe the responses of the two modes independently after the coupling modulation in Eq. (10). The last term describes the responses of cross coupling between the two modes after coupling modulation. The fitted reflection spectra based on Eq. (11) are depicted as green olive-colored solid curves $(d=0 \mathrm{~nm})$, red wine-colored dash-dot curves $(d=40 \mathrm{~nm})$, and navy short-dash curves $(d=80$ $\mathrm{nm}$ ), respectively. In Figure 3, for both the $x$ and $y$ polarizations, the simulated and fitted results for both the 
reflection intensity spectra and phase spectra are consistent. Figure 5 reports the curve of the fitted parameters $\omega_{\alpha}, \gamma_{\alpha}(\alpha=$ $p, q)$ with variations in asymmetry degree $d$. The radiation loss of the two polarized dressed bright modes decreased as $d$ increased, whereas the radiation loss of the dressed dark modes increased with $d$. Moreover, as the asymmetry degree $d$ increased, coupling between the two modes improved, and the bright mode transferred energy gradually to the dressed dark mode. As reported in Figure 5(b, d), the circular frequencies of the two dressed modes were widely distributed with an increase in the asymmetry degree for the $x$-polarization. However, for the $y$-polarization, the circular frequency of the dressed mode was more narrowly distributed because the frequency difference between the two modes was $11.7 \mathrm{THz}$ for the $x$-polarization and $41.8 \mathrm{THz}$ for the $y$-polarization. Compared with the $y$-polarization, the effective coupling coefficient $\mathrm{K}=\left|\sqrt{\gamma_{p} \gamma_{q}} /\left(\omega_{q}-\omega_{p}\right)\right|$ for the $x$-polarization was substantially higher [20], resulting in a wider distribution of circular frequencies.
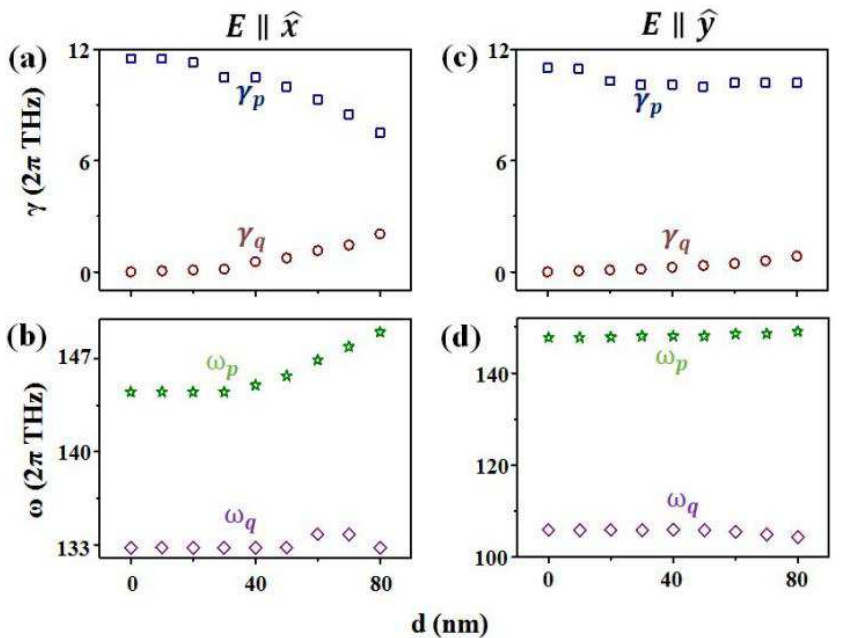

Figure 5. (Color online) Variation curves of $\gamma_{p}$ (navy open squares), $\gamma_{q}$ (red wine-colored open circles) with $d$ for the x-polarization (a) and $y$-polarization (c) fitted to the simulated reflectance spectra drawn from Eq. (10). For the x-polarization (b) and y-polarization (d), the curves of $\omega_{p}$ (green olive-colored open stars) and $\omega_{q}$ (red wine-colored open diamonds) were drawn from the fitted simulated reflectance spectra with d according to Eq. (11).

\section{Conclusions}

In this study, A sandwich-type metasurface of $\mathrm{Au} / \mathrm{VO}_{2} / \mathrm{Au}$ in a near-infrared band was presented. Through combining the phase transition of $\mathrm{VO}_{2}$ and the symmetry-breaking of a nanoparticle with a square hole, the following optical properties of the metasurface were observed: (1) For the same polarization and structure, an efficient switching function was achieved by tuning the phase transition of $\mathrm{VO}_{2}$; (2) a substantial change in reflection intensity was achieved by adjusting the percentage of the semiconductor phase of $\mathrm{VO}_{2}$; (3) independent modification of the phase from $<180^{\circ}$ to nearly $360^{\circ}$ was achieved by adjusting the asymmetry or polarization of the structure; (4) consequently, an independent regulation of amplitude and phase was accomplished.
Through providing a theoretical explanation of these results through two-mode and one-port tCMT, the conclusion indicated that mode coupling was substantially modulated during the reflection phase, and phase transition of $\mathrm{VO}_{2}$ was substantially modulated during the reflection amplitude. The research findings may be applied to the design of multifunctional devices, especially gradient metasurfaces and temperature-controlled switches. Furthermore, the phenomenon observed through the modulation of the degree of symmetry-breaking provides a novel design concept for the realization of independently modulating electromagnetic waves.

\section{Acknowledgements}

The project supported by the National Natural Science Foundation of China (NSFC; 11304002), the Natural Science Foundation of Anhui Province 1208085MA07), and the Anhui Provincial Quality Project for Higher Education Institutions (Grant Nos. 2019mooc066). This manuscript was edited by Wallace Academic Editing.

\section{References}

[1] Liu, M., Hwang, H. Y., Tao, H., Strikwerda, A. C., Fan, K., Keiser, G. R., Sternbach, A. J., West, K. G., Kittiwatanakul, S., Lu, J., Wolf, S. A., Omenetto, F. G., Zhang, X., Nelson, K. A., \& Averitt, R. D. (2012) Terahertz-field-induced insulator-to-metal transition in vanadium dioxide metamaterial. Nature, 487, 345-348.

[2] Aetukuri, N. B., Gray, A. X., Drouard, M., Cossale, M., Gao, L., Reid, A. H., Kukreja, R., Ohldag, H., Jenkins, C. A., Arenholz, E., Roche, K. P., Dürr, H. A., Samant, M. G., \& Parkin, S. S. P. (2013) Control of the metal-insulator transition in vanadium dioxide by modifying orbital occupancy. Nat. Phys. 9, 661-666.

[3] Budai, J. D., Hong, J., Manley, M. E., Specht, E. D., Li, C. W., Tischler, J. Z., Abernathy, D. L., Said, A. H., Leu, B. M., Boatner, L. A., McQueeney, R. J., \& Delaire, O. (2014) Metallization of vanadium dioxide driven by large phonon entropy. Nature 515, 535-539.

[4] Lee, S., Hippalgaonkar, K., Yang, F., Hong, J., Ko C., Suh, J., Liu, K., Wang, K., Urban, J. J., Zhang, X., Dames, C., Hartnoll, S. A., Delaire, O., \& Wu, J. (2017) Anomalously low electronic thermal conductivity in metallic vanadium dioxide. Science 355, 371-374.

[5] Xu, G., Chen, Y., Tazawa, M., \& Jin, P. (2006) Surface Plasmon Resonance of Silver Nanoparticles on Vanadium Dioxide. J. Phys. Chem. B 110, 2051-2056.

[6] Wei, J., Wang, Z., Chen, W., \& Cobden, D. H. (2009) New aspects of the metal-insulator transition in single-domain vanadium dioxide nanobeams. Nat. Nanotechnol. 4, 420-424.

[7] Zhu, H., Zhang, Y., Ye, L., Li, Y., Xu, Y., \& Xu, R. (2020) Switchable and tunable terahertz metamaterial absorber with broadband and multi-band absorption. Opt. Express 26: 38626. 
[8] Driscoll, T., Palit, S., Qazilbash, M. M., Brehm, M., Keilmann, F., Chae B., Yun, S., Kim, H., Cho, S. Y., Jokerst, N. M., Smith, D. R., \& Basov, D. N. (2008) Dynamic tuning of an infrared hybrid-metamaterial resonance using vanadium dioxide. Appl. Phys. Lett. 93, 024101.

[9] Huang, W., Yin, X., Huang, C., Wang, Q., Miao, T., \& Zhu, Y (2010) Optical switching of a metamaterial by temperature controlling. Appl. Phys. Lett. 96, 261908.

[10] Ooi, K. J. A., Bai, P., Chu, H. S., \& Ang, L. K. (2013) Ultracompact vanadium dioxide dual-mode plasmonic waveguide electroabsorption modulator. Nanophotonics 2, 13-19.

[11] Nouman, M. T., Hwang, J. H., Faiyaz, M., Lee, K. J., Noh, D. Y., \& Jang, J. H. (2018) Vanadium dioxide based frequency tunable metasurface filters for realizing reconfigurableterahertz optical phase and polarization control. Opt. Express 26, 12922-12929.

[12] Wang, T., He, J., Guo, J., Wang, X., Feng, S., Kuhl, F., Becker, M., Polity, A., Klar, P. J., \& Zhang, Y. (2019) Thermally switchable terahertz wavefront metasurface modulators based on the insulator-to-metal transition of vanadium dioxide. Opt. Express 27, 20347-20357.

[13] Qu, C., Ma, S., Hao, J., Qiu, M., Li, X., Xiao, S., Miao, Z., Dai, N., He, Q., Sun, S., \& Zhou, L. (2015) Tailor the Functionalities of Metasurfaces Based on a Complete Phase Diagram. Phys. Rev. Lett. 115, 235503.

[14] Miao, Z., Wu, Q., Li, X., He, Q., Ding, K., An, Z., Zhang, Y., \& Zhou, L. (2015) Widely Tunable Terahertz Phase Modulation with Gate-Controlled Graphene Metasurfaces. Phys. Rev. X 5, 041027.

[15] Ozbay, E. (2006) Plasmonics: Merging Photonics and Electronics at Nanoscale Dimensions. Science 311, 189-193.
[16] Huang, W., Zhang, X., Wang, Q., Wang, M., Li, C., Li, K., Yang, X., \& Shi J. (2019) Controllability of surface plasmon polariton far-field radiation using a metasurface. Photonics Res. 7, 728-733.

[17] Verleur, H. W., Barker, A. S., \& Berglund, C. N. (1968) Optical Properties of $\mathrm{VO}_{2}$ between 0.25 and $5 \mathrm{eV}$. Phys. Rev. $172,788-798$

[18] Lv, T. T., Li, Y. X., Ma, H. F., Zhu, Z., Li, Z. P., Guan, C. Y., Shi, J. H., Zhang, H., \& Cui, T. J. (2016) Hybrid metamaterial switching for manipulating chirality based on $\mathrm{VO}_{2}$ phase transition. Sci. Rep. 6, 23186.

[19] Agranovich, V. M., \& Kravtsov, V. E. (1985) Notes on crystal optics of superlattices. Solid State Commun. 55, 85-90.

[20] Huang, W., Lin, J., Qiu, M., Liu, T., He, Q., Xiao, S., \& Zhou, L. (2020) A complete phase diagram for dark-bright coupled plasmonic systems: applicability of Fano's formula. Nanophotonics 9, 3251-3262.

[21] Miroshnichenko, A. E., Flach, S., \& Kivshar, Y. S. (2010) Fano resonances in nanoscale structures. Rev. Mod. Phys. 82, 2257-2298.

[22] Fan, S., Suh W., \& Joannopoulos, J. D. (2003) Temporal coupled-mode theory for the Fano resonance in optical resonators. J. Opt. Soc. Am. A 20, 569-572.

[23] Lin, J., Qiu, M., Zhang, X., Guo, H., Cai, Q., Xiao, S., He, Q., \& Zhou, L. (2020) Tailoring the lineshapes of coupled plasmonic systems based on a theory derived from first principles. Light Sci. Appl. 9, 158.

[24] Huang, W., Zhao, G., Guo, J., Wang, M., \& Shi, J. (2016) Nearly Perfect Absorbers Operating Associated with Fano Resonance in the Infrared Range. Chin. Phys. Lett. 33, 088103. 